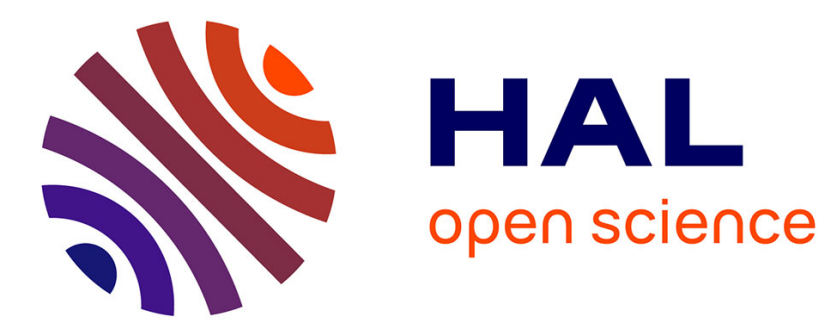

\title{
Effective capacity based resource allocation for Rayleigh-fading parallel channels
}

\author{
Philippe Ciblat, Ivan Stupia, Luc Vandendorpe
}

\section{To cite this version:}

Philippe Ciblat, Ivan Stupia, Luc Vandendorpe. Effective capacity based resource allocation for Rayleigh-fading parallel channels. 2018 IEEE 19th International Workshop on Signal Processing Advances in Wireless Communications (SPAWC), Jun 2018, Kalamata, Greece. pp.1-5, 10.1109/spawc.2018.8445897 . hal-02439516

\section{HAL Id: hal-02439516 https://hal.science/hal-02439516}

Submitted on 14 Jan 2020

HAL is a multi-disciplinary open access archive for the deposit and dissemination of scientific research documents, whether they are published or not. The documents may come from teaching and research institutions in France or abroad, or from public or private research centers.
L'archive ouverte pluridisciplinaire HAL, est destinée au dépôt et à la diffusion de documents scientifiques de niveau recherche, publiés ou non, émanant des établissements d'enseignement et de recherche français ou étrangers, des laboratoires publics ou privés. 


\title{
Effective capacity based resource allocation for Rayleigh-fading parallel channels
}

\author{
Philippe Ciblat*, Ivan Stupia ${ }^{\dagger}$, Luc Vandendorpe ${ }^{\dagger}$ \\ *Telecom ParisTech, Université Paris-Saclay, Paris, France, ciblat@telecom-paristech.fr \\ †Université catholique de Louvain, Louvain-la-Neuve, Belgium, ivan.stupia@uclouvain.be, luc.vandendorpe@uclouvain.be
}

\begin{abstract}
We address the problem of allocating different powers amongst parallel channels when effective capacity is the performance metric and sum-power is constrained. We assume that Chase-Combining-HARQ mechanism is applied. Closedform expressions for the powers are exhibited. Numerical comparisons with other power allocations obtained through either ergodic capacity or throughput optimizations are done.

Index Terms - Effective capacity, Delay-constrained communications, Resource allocation, Statistical CSI
\end{abstract}

\section{INTRODUCTION}

In many applications (video streaming, voice, vehicle to vehicle communications), stringent delay has to be fulfilled and resource allocation has to be done accordingly [1]. One way to take into account the Quality of Service devoted to delay is the use of the so-called effective capacity encompassing from the ergodic capacity (infinite delay) to the delay-limited capacity (zero delay). More precisely the effective capacity introduced in [2] represents the achievable data rate given a parameter $\theta$ indicating the exponential delay rate of the buffer overflow probability (which may be related to the delay due to the Little's law [3]).

In the literature, the papers related to effective capacity are twofold: on the hand, they offer some simplified or exact expressions for the effective capacity under certain asymptotic regimes as in [4], [5] or compare numerically the effective capacity of some practical schemes as in [6]. On the other hand, they are devoted to resource allocation with effective capacity as the performance metric. In [7], [8], [9], the power is optimized for different network configurations but assuming perfect Channel State Information at the Transmitter (CSIT). Rate (of the first transmitted packet within a Hybrid ARQ mechanism) can be also optimized as in [11] for a multicast environment or for different traffic models as in [10]. Notice that the rate optimization is done through a computational manner without exact closed-form expression. In [12], the channel assignment and power optimization are analyzed but only sub-optimal solutions are provided in the general case for channel assignment and optimal power of the users are provided by numerical solutions assuming no sum-power constraint. One can remark that only recent attention has been paid on this metric for resource allocation and so few problems have been solved and often with the help of approximations.

In this paper we propose to share optimally a predefined amount of power among parallel Rayleigh fading channels

This work was supported by the ERC Starting Grant "CTO Com". when only statistical CSIT is available and when the sum effective capacity is the objective function. In other words, we would like to mimic the waterfilling algorithm when the effective capacity is considered instead of the instantaneous Shannon's capacity.

The rest of the paper is organized as follows: Section II is devoted to the system model. In Section III, we describe mathematically our optimization problem and provide some interesting properties. In Section IV, we solve our optimization problem and propose also other power distributions when more standard objective functions are considered such as either the ergodic capacity or the throughput. Numerical illustrations are provided in Section V. In Section VI, concluding remarks are drawn.

\section{NETWORK AND SYSTEM MODEL}

We consider $K$ slotted parallel communication channels. On each channel, let's say $k$, one user is assigned and encounters a Rayleigh flat fading channel whose the output/input equation for the slot $\ell$ is

$$
\mathbf{y}_{k}(\ell)=h_{k}(\ell) \mathbf{x}_{k}(\ell)+\mathbf{w}_{k}(\ell)
$$

where $\mathbf{y}_{k}(\ell), \mathbf{x}_{k}(\ell)$, and $\mathbf{w}_{k}(\ell)$ are the received samples, the transmitted symbols, and the noise vector for the slot $\ell$ respectively. In addition, we assume that

- $h_{k}(\ell)$ is the random process related to the channel. The process $h_{k, \ell}$ is i.i.d. with respect to (wrt) $\ell$, and only independent (but not i.d.) wrt $k$. So $h_{k}(\ell)$ is a zero-mean circularly-symmetric Gaussian random variable with variance $\sigma_{k}^{2}=\mathbb{E}\left[g_{k}(\ell)\right]$ where $g_{k}(\ell)$ denotes $\left|h_{k}(\ell)\right|^{2}$.

- $\mathbf{w}_{k}(\ell)$ is a zero-mean circularly symmetric white Gaussian vector with variance $N_{0}$ per component.

- $P_{k}$ is the power used by the user $k$ for sending one component of its vector $\mathbf{x}_{k}(\ell)$. The power is independent of $\ell$ since it will be optimized only with the knowledge of the statistical CSIT, i.e., of $\left\{\sigma_{k}^{2}\right\}_{k=1}^{K}$.

The effective capacity for user $k$, denoted by $\mathcal{C}_{k}$, is equal to

$$
\mathcal{C}_{k}=\lim _{\ell \rightarrow \infty} \frac{1}{\theta_{k} \ell} \log \left(\mathbb{E}\left[e^{-\theta_{k} N_{\ell}}\right]\right)
$$

with

- $N_{\ell}$ the number of correctly received packets at slot $\ell$,

- $\theta_{k}$ the so-called statistical QoS requirement defined as

$$
\theta_{k}=\lim _{q \rightarrow \infty} \frac{-\log \operatorname{Pr}\left(Q_{k} \geq q\right)}{q}
$$


where $Q_{k}$ is the queue length for user $k$ and $q$ is the overflow threshold [2], [5], [10].

Actually $\theta_{k}$ controls the exponential decay of the buffer overflow probability. So when $\theta_{k} \rightarrow 0$, there is no delay constraint since the queue length can be arbitrarily large, and thus the effective capacity tends to the ergodic capacity. Conversely, when $\theta_{k} \rightarrow \infty$, the delay is forced to be arbitrary small since the queue has to be flushed as soon as possible, and thus the effective capacity tends to the delay-limited capacity. Therefore $\theta_{k}$ is inversely related to the statistical delay constraint.

In addition, an HARQ mechanism is carried out. In this paper, a Chase-Combining HARQ (CC-HARQ) is implemented [13]. More precisely, an information packet coming from the upper layer is encoded into a slot with the rate $R_{k}(\mathrm{bits} / \mathrm{s} / \mathrm{Hz})$. If this slot is not correctly received at the receiver side, NonAcknowledgment (NACK) is sent back, and then the packet sent during the previous packet is transmitted again. Finally a Maximum Ratio Combiner on both received packets is applied, and so on.

We assume we have $K$ parallel channels whose each of which carries a CC-HARQ mechanism with a different statistical delay constraint. In addition, the sum power is shared among the $K$ parallel channels. Consequently, we focus on the following optimization problem.

Problem 1. Maximization of the sum effective capacity with respect to $\left\{P_{k}\right\}_{k=1}^{K}$ with $P_{k}$ the power of user/channel $k$ leads to the following problem

$$
\begin{aligned}
{\left[P_{1}^{\star}, \ldots, P_{K}^{\star}\right]=} & \arg \max _{P_{1}, \ldots, P_{K}} \sum_{k=1}^{K} \mathcal{C}_{k}\left(P_{k}\right) \\
\text { s.t. } \quad & \sum_{k=1}^{K} P_{k}=P_{\max } \\
& P_{k} \geq 0, \forall k .
\end{aligned}
$$

According to [5], the effective capacity for CC-HARQ, when the number of transmissions is infinite, is

$$
\mathcal{C}_{k}\left(P_{k}\right)=R_{k}+\frac{1}{\theta_{k}}\left(\frac{\alpha_{k}}{P_{k}}-W_{0}\left(\frac{\alpha_{k} \beta_{k}}{P_{k}} \cdot e^{\frac{\alpha_{k}}{P_{k}}}\right)\right)
$$

with $\alpha_{k}=N_{0}\left(2^{R_{k}}-1\right) / \sigma_{k}^{2}>0, \beta_{k}=e^{\theta_{k} R_{k}}>1$, and $W_{0}$ the W-Lambert function. Notice that Incremental-Redundancy HARQ is not treated since there is no closed-form expression as Eq. (4) for $\mathcal{C}_{k}$.

\section{OPTIMIZATION PROBLEM PROPERTY}

We will prove that the optimization Problem 1 is convex. As the constraints are linear, the feasible set is convex. So we just have to show that the objective function is concave in $\left\{P_{k}\right\}_{k=1}^{K}$ (since it is a maximization problem and not a minimization problem). By removing the terms independent of $P_{k}$ in $\mathcal{C}_{k}$, we just need to prove the concavity of these following functions

$$
f_{k}: x \mapsto \frac{1}{\theta_{k}}\left(\frac{\alpha_{k}}{x}-W_{0}\left(\frac{\alpha_{k} \beta_{k}}{x} \cdot e^{\frac{\alpha_{k}}{x}}\right)\right) .
$$

Let $W_{0}^{\prime}$ be the derivative function of $W_{0}$. As $W_{0}^{\prime}(x)=$ $W_{0}(x) /\left(x\left(1+W_{0}(x)\right)\right)$, we obtain easily the derivative of $f_{k}$ as

$$
f_{k}^{\prime}(x)=\frac{1}{\theta_{k} x} \cdot \frac{W_{0}\left(\frac{\alpha_{k} \beta_{k}}{x} \cdot e^{\frac{\alpha_{k}}{x}}\right)-\frac{\alpha_{k}}{x}}{1+W_{0}\left(\frac{\alpha_{k} \beta_{k}}{x} \cdot e^{\frac{\alpha_{k}}{x}}\right)},
$$

and the second-derivative of $f_{k}$ as

$$
f_{k}^{\prime \prime}(x)=\frac{\alpha_{k} /\left(x^{3} \theta_{k}\right)}{1+\omega_{k}(x)}\left(2-\frac{\omega_{k}(x)}{\alpha_{k} / x}-\frac{\frac{\omega_{k}(x)}{\alpha_{k} / x}}{\left(\frac{1+\omega_{k}(x)}{1+\alpha_{k} / x}\right)^{2}}\right)
$$

with $\omega_{k}(x)=W_{0}\left(\frac{\alpha_{k} \beta_{k}}{x} \cdot e^{\frac{\alpha_{k}}{x}}\right)$ which is non-negative as $\omega_{k}(x) \geq \alpha_{k} / x$ for $\beta_{k}>1$. By setting $y=\omega_{k}(x)$ and $z=\alpha_{k} / x$, proving the negativity of $f_{k}^{\prime \prime}$ for any $\alpha_{k}$ and $\beta_{k}$ is equivalent to proving the negativity of

$$
g(y, z)=2-\frac{y}{z}-\frac{y / z}{((1+y) /(1+z))^{2}} .
$$

for any arbitrary positive values of $y$ and $z$ but with $y>z$.

By setting $\gamma=y / z$, we wish to show the negativity of

$$
z \mapsto h_{\gamma}(z)=2-\gamma-\frac{\gamma}{((1+z \gamma)(1+z))^{2}}
$$

for any $\gamma>1$ and any positive $z$, and it is equivalent to show the negativity of the parametrized second-order polynomial

$$
z \mapsto \tilde{h}_{\gamma}(z)=2(1-\gamma)+2 \gamma(1-\gamma) z+\left(2 \gamma^{2}-\gamma-\gamma^{3}\right) z^{2} .
$$

As $2 \gamma^{2}-\gamma-\gamma^{3}<0$ for any $\gamma>1$, the polynomial $z \mapsto$ $\tilde{h}_{\gamma}(z)$ can be positive only between two roots $r_{1}$ and $r_{2}$. Its discriminant is equal to $\Delta=-4 \gamma(\gamma-1)^{2}(\gamma-2)$. Therefore we have two cases:

- If $\gamma>2$, then $\Delta<0$ and the polynomial $z \mapsto \tilde{h}_{\gamma}(z)$ does not have real-valued roots and so is always negative. Consequently $g(y, z)$ is also negative and the function $f_{k}$ is concave.

- If $\gamma \leq 2$, then $\Delta \geq 0$ and the polynomial $z \mapsto \tilde{h}_{\gamma}(z)$ has two real-valued roots:

$$
r_{1}=\frac{(\gamma-1)(\gamma-\sqrt{2-\gamma})}{2 \gamma^{2}-\gamma-\gamma^{3}}
$$

and

$$
r_{2}=\frac{(\gamma-1)(\gamma+\sqrt{2-\gamma})}{2 \gamma^{2}-\gamma-\gamma^{3}}
$$

As $2 \gamma^{2}-\gamma-\gamma^{3}<0$, it is easy to check that $r_{1}$ and $r_{2}$ are negative. Consequently $z \mapsto \tilde{h}_{\gamma}(z)$ is negative for any value of $z>0$, and the function $f_{k}$ is concave.

As a conclusion, Problem 1 is convex and will be solved analytically in next section. 


\section{POWER OPTIMIZATION ALGORITHM}

As Problem 1 is convex, we can apply the Karush-KuhnTucker (KKT) conditions [17] on the following problem

$$
\left[P_{1}^{\star}, \ldots, P_{K}^{\star}\right]=\arg \max _{\left\{P_{1}, \ldots, P_{K}\right\}} \sum_{k=1}^{K} f_{k}\left(P_{k}\right)
$$

such that Eqs. (2)-(3) are satisfied.

The below-written derivations are based on those of [14] focusing on power allocation for capacity with any arbitrary input distributions. The difference lies in the expression of functions $f_{k}$. The Lagrangian function is

$$
\mathcal{L}\left(P_{1}, \ldots, P_{K}, \lambda\right)=-\sum_{k=1}^{K} f_{k}\left(P_{k}\right)+\lambda\left(\sum_{k=1}^{K} P_{k}-P_{\max }\right)
$$

with $\lambda \geq 0$ the Lagrangian multiplier. Then the KKT conditions lead to

$$
\begin{aligned}
-f_{k}^{\prime}\left(P_{k}\right)+\lambda=-\frac{1}{\alpha_{k} \theta_{k}} g_{\beta_{k}}\left(\alpha_{k} / P_{k}\right)+\lambda & =0, \quad \forall k \\
\lambda\left(\sum_{k=1}^{K} P_{k}-P_{\max }\right) & =0
\end{aligned}
$$

with

$$
g_{\beta}(x)=x \cdot \frac{W_{0}\left(\beta x e^{x}\right)-x}{1+W_{0}\left(\beta x e^{x}\right)} .
$$

As one can prove that the image of $f_{k}^{\prime}$ is $\left[0, \log \left(\beta_{k}\right) /\left(\alpha_{k} \theta_{k}\right)\right]$ or equivalently the image of $g_{\beta_{k}}$ is $\left[0, \log \left(\beta_{k}\right)\right]$, it turns out that

$$
\begin{cases}\log \left(\beta_{k}\right)<\alpha_{k} \theta_{k} \lambda & \Rightarrow P_{k}^{\star}=0 \\ \text { else } & \Rightarrow P_{k}^{\star}=\frac{\alpha_{k}}{g_{\beta_{k}}^{(-1)}\left(\alpha_{k} \theta_{k} \lambda\right)}\end{cases}
$$

with $g_{k}^{(-1)}$ the inverse function of $g_{k}$. As, by convention, $g_{\beta_{k}}^{(-1)}\left(\log \left(\beta_{k}\right)\right)=+\infty$ we have the following main result.

Result 1. The solution of Problem 1 is

$$
P_{k}^{\star}=\frac{\alpha_{k}}{g_{\beta_{k}}^{(-1)}\left(\min \left(\alpha_{k} \theta_{k} \lambda^{\star}, \log \left(\beta_{k}\right)\right)\right)}, \forall k
$$

with $\lambda^{\star}$ the water level such that $\sum_{k=1}^{K} P_{k}^{\star}=P_{\max }$.

In order to find out numerically $\left\{P_{k}^{\star}\right\}_{k=1}^{K}$, we need to resort to a Look Up Table (LUT) on the set of functions $x \mapsto g_{\beta_{k}}^{(-1)}(x)$. To overcome this potential issue, Problem 1 can be solved

- using standard numerical tools adapted to convex optimization (e.g., interior-point method), or

- using approximation of $x \mapsto g_{\beta_{k}}^{(-1)}(x)$ as done below. In [15], the following series expansion in $p$ for the $\mathrm{W}$ Lambert function is given

$$
W_{0}\left(q e^{q} e^{p}\right)=q+\frac{q}{1+q} p+\ldots
$$

which leads to

$$
g_{\beta_{k}}(x) \approx \widetilde{g}_{k}(x) \text { with } \widetilde{g}_{k}(x)=\frac{x^{2} \log \left(\beta_{k}\right)}{(1+x)^{2}+x \log \left(\beta_{k}\right)} .
$$

We now want to inverse $\widetilde{g}_{k}$. Let $\delta_{k}=\log \left(\beta_{k}\right)$. After tedious but simple algebraic manipulations, we obtain, for $y \in\left[0, \delta_{k}\right]$, that

$$
\tilde{g}_{k}^{(-1)}(y)=\frac{y\left(2+\delta_{k}\right)+\sqrt{\left(y \delta_{k}+4 y+4\right) y \delta_{k}}}{2\left(\delta_{k}-y\right)} .
$$

Consequently the approximate solution of Problem 1 is given by

$$
{\widetilde{P_{k}}}^{*}=\frac{2 \alpha_{k} \min \left(\delta_{k}-\alpha_{k} \theta_{k} \widetilde{\lambda}^{\star}, 0\right)}{m_{k}\left(2+\delta_{k}\right)+\sqrt{s_{k}}}
$$

where $s_{k}=\left(m_{k} \delta_{k}+4 m_{k}+4\right) m_{k} \delta_{k}$ with $m_{k}=$ $\min \left(\alpha_{k} \theta_{k} \widetilde{\lambda}^{\star}, \delta_{k}\right)$ and $\widetilde{\lambda}^{\star}$ chosen such that $\sum_{k=1}^{K}{\widetilde{P_{k}}}^{\star}=$ $P_{\max }$.

We also propose to compare the previously-obtained power optimization to those obtained by maximizing more standard performance metrics such as sum ergodic capacity and sumthroughput.

Ergodic capacity based power optimization: Whereas the ergodic capacity is achieved by the optimal coding scheme, we cannot set $\theta_{k} \rightarrow 0$ for any $k$ in previously-described derivations since we have forced the use of the not-optimal CC-HARQ mechanism. This can be easily checked by remarking that the limit of Eq. (4) over $\theta_{k}$ is different from the ergodic capacity by using the series expansion provided by Eq. (9). The sum ergodic capacity optimization problem over the powers can be written as

$$
\left[P_{1}^{\star, \text { ergo }}, \ldots, P_{K}^{\star, \text { ergo }}\right]=\arg \max _{\left\{P_{1}, \ldots, P_{K}\right\}} \sum_{k=1}^{K} h_{k}\left(P_{k}\right)
$$

such that Eqs. (2)-(3) are satisfied, and where $h_{k}$ is the ergodic capacity of the user $k$ given by [19]

$$
h_{k}(x)=e^{\nu_{k} / x} E_{1}\left(\nu_{k} / x\right)
$$

with $E_{1}$ the integral exponential [20] and $\nu_{k}=N_{0} / \sigma_{k}^{2}$.

This problem is convex and, following the same approach as above, the KKT conditions lead to

$$
P_{k}^{\star, \text { ergo }}=h_{k}^{(-1)}\left(\min \left(\lambda^{\star, \text { ergo }}, 1 / \nu_{k}\right)\right)
$$

where $h_{k}^{\prime(-1)}$ is the inverse function of $h_{k}^{\prime}$ given by

$$
h_{k}^{\prime}(x)=\frac{1}{x}-\frac{\nu_{k}}{x^{2}} e^{\nu_{k} / x} E_{1}\left(\nu_{k} / x\right)
$$

and $\lambda^{\star, \text { ergo }}$ the water level such that $\sum_{k=1}^{K} P_{k}^{\star, \text { ergo }}=P_{\max }$.

Once again the numerical evaluation of the optimal powers can be done through LUT or approximation for $h_{k}^{\prime(-1)}$, i.e., for $E_{1}$. Discussion about approximation is out of the scope of the paper since this optimization has just be done for comparison purpose. 
Throughput based power optimization: According to [16], when CC-HARQ with infinite retransmission number is assumed, the throughput of user $k$ can be written as

$$
\eta_{k}=R_{k} \frac{1}{1+\frac{\alpha_{k}}{P_{k}}}
$$

Eq. (10) was available in [18] but as an approximation of the throughput for practical coding scheme. Here the derivations are exact and done by using achievable capacity coding scheme and outage probability. The sum-throughput optimization problem over the powers is

$$
\left[P_{1}^{\star, t h r u}, \ldots, P_{K}^{\star, t h r u}\right]=\arg \max _{\left\{P_{1}, \ldots, P_{K}\right\}} \sum_{k=1}^{K} j_{k}\left(P_{k}\right)
$$

such that Eqs. (2)-(3) are satisfied, and where

$$
j_{k}(x)=R_{k} \frac{1}{1+\frac{\alpha_{k}}{x}} .
$$

This problem is still convex and, following the same approach as above, KKT conditions lead to

$$
P_{k}^{\star, t h r u}=\sqrt{\left.\frac{R_{k} \alpha_{k}}{\min \left(\lambda^{\star}, t h r u\right.}, R_{k} / \alpha_{k}\right)}-\alpha_{k}
$$

with $\lambda^{\star, t h r u}$ the water level such that $\sum_{k=1}^{K} P_{k}^{\star, t h r u}=P_{\max }$.

\section{NUMERICAL RESULTS}

We consider two configurations with $K=10$.

- Configuration 1: $\sigma_{k}^{2} / N_{0}=k, R_{k}=k$, and $\theta_{k}=1$. The delay constraint is moderate and the coding rate of the first packet increases accordingly to the channel quality.

- Configuration 2: $\sigma_{k}^{2} / N_{0}=k, R_{k}=k$, and $\theta_{k}=k$. Compared to Configuration 1, the delay constraint increases too accordingly to the channel quality. Notice that power allocation for both configurations will be identical when optimizing throughput and ergodic capacity since they are independent of $\theta_{k}$.

In Fig. 1, we plot the powers per channel obtained by the four optimization algorithms $\left(P_{\max }=10\right)$. We observe that the power allocation is different and so is dependent on the considered metric for optimization as well as on the considered configuration. Except when the ergodic capacity (which is not upper-bounded whatever $\theta_{k}$ and $R_{k}$ ) is considered, some channels are not used since the unassigned channels are too expensive for their respective gains. We also note that the stronger the delay constraint is, the more different the power allocation related the effective capacity optimization compared to those obtained with the throughput optimization is.

In Fig. 2, we plot the sum effective capacity vs. $P_{\max }$ for the four power allocations obtained by optimizing the throughput, the ergodic capacity, the effective capacity, and the approximate effective capacity respectively. Obviously the best performance is obtained by considering the powers provided through the optimization of the effective capacity. The loss in performance with other power allocations is significant for considered configurations except when we compare the

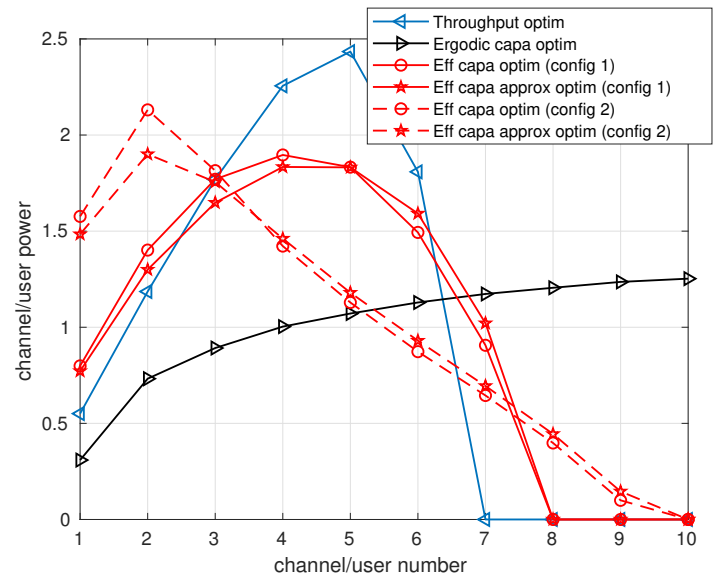

Fig. 1. Optimized powers vs. users

throughput based allocation to the effective capacity based allocation in Configuration 1. Indeed, in Configuration 1, the delay constraint is quite weak and the most important phenomenon is the rate-limited transmission (according to $R_{k}$ ) which acts almost in the same way for the throughput and effective capacity criteria (see also the power allocation obtained for both criteria in Configuration 1 on Fig. 1).

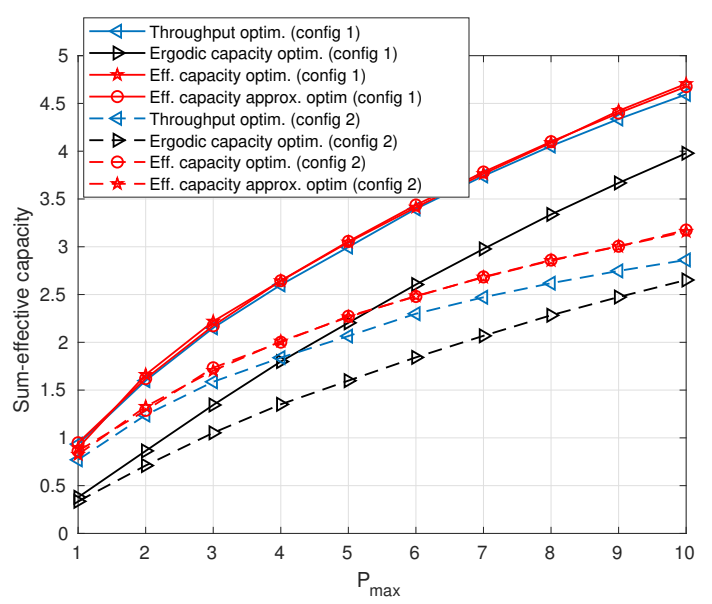

Fig. 2. Sum effective capacity vs. $P_{\max }$

\section{CONCLUSION}

We have found the power distribution optimizing the sum effective capacity for Rayleigh-fading parallel channels. Further investigations may be done: i) the adaptation of the rate $R_{k}$ in addition to the power, but we have checked that we boil down a non-convex optimization problem since $\left(P_{k}, R_{k}\right) \mapsto \bar{f}_{k}\left(P_{k}, R_{k}\right)$ obtained by seeing $R_{k}$ as a variable in Eq. (5) is not jointly convex but $P_{k} \mapsto \bar{f}_{k}\left(P_{k}, R_{k}^{\star}\left(P_{k}\right)\right)$ seems to be convex where $R_{k}^{\star}\left(P_{k}\right)=\arg \max _{R} \bar{f}_{k}\left(P_{k}, R\right)$ but we did not manage to prove it, ii) the extension to Incremental Redundancy (IR)-HARQ, but the difficulty lies in the absence of closed-form expression for the effective capacity. 


\section{REFERENCES}

[1] C. She, C. Yang, and T.Q.S. Quek, "Radio resource management for ultra-reliable and low latency communications," IEEE Communications Magazine, vol. 55, no. 6, pp. 72-78, June 2017.

[2] D. Wu, R. Negi, "Effective capacity: a wireless link model for support of quality of service," IEEE Transactions on Wireless Communications, vol. 2, no. 4, pp. 630-643, July 2003.

[3] J.D.C. Little, "A proof of the queuing formula $L=\lambda W$," Operations Research, vol. 9, no. 30, pp. 383-387, March 1961.

[4] Y. Li, M.C. Gursoy, and S. Velipasalar, "On the throughput of HybridARQ under QoS constraints," IEEE Transactions on Vehicular Technology, vol. 64, no. 6, pp. 2725-2732, June 2015.

[5] P. Larsson, J. Gross, H. Al-Zubaidy, L. Rasmussen, and M. Skoglund, "Effective capacity of retransmission schemes - a recurrence relation approach," IEEE Transactions on Communications, vol. 64, no. 11, pp. 4817-4835, November 2016.

[6] R. Sassioui, L. Szczecinski, L. Le, and M. Benjillali, "AMC and HARQ: effective capacity analysis," IEEE Wireless Communications and Networking Conference (WCNC), Doha (Qatar), April 2016.

[7] W. Yu, L. Musavian, and Q. Ni, "Statistical Delay QoS driven Energy Efficiency and Effective Capacity Tradeoff for uplink multi-user multicarrier systems," IEEE Transactions on Communications, to be published in 2017 (early access).

[8] J. Tang, X. Zhang, "Quality of Service driven power and rate adaptation over wireless links," IEEE Transactions on Wireless Communications, vol. 6, no. 8, pp. 3058-3068, August 2008.

[9] D. Qiao, M.C. Gursoy, and S. Velipasalar, "Effective capacity region and optimal power control for fading broadcast channels," IEEE International Symposium on Information Theory (ISIT), Saint-Petersburg (Russia), July 2011.

[10] Y. Li, G. Ozcan, M.C. Gursoy, and S. Velipasalar, "Energy efficiency of Hybrid-ARQ under statistical queuing constraints," IEEE Transactions on Communications, vol. 64, no. 10, pp. 4253-4267, October 2016.

[11] Q. Du, X. Zhang, "Effective capacity optimization with layered transmission for multicast in wireless networks," IEEE International Wireless Communications and Mobile Computing (IWCMC), Crete Island (Greece), August 2008.

[12] D. To, H.X. Nguyen, Q.-T. Vien, and L.-K. Huang, "Power allocation for HARQ-IR systems under QoS constraints and limited feedback," IEEE Transactions on Wireless Communications, vol. 14, no. 3, pp. 1581-1594, March 2015.

[13] S. Lin, D.J. Costello, and M.J. Miller "Automatic-repeat-request error control schemes," IEEE Communications Magazine, vol. 22, no. 12, pp. 5-16, December 1984.

[14] A. Lozano, A.M. Tulino, and S. Verdú, "Optimum power allocation for parallel Gaussian channels with arbitrary input distributions," IEEE Transactions on Information Theory, vol. 52, no. 7, pp. 3033-3051, July 2006.

[15] R.M. Corless, D.J. Jeffrey, and D.E. Knuth, "A sequence of series for the Lambert W function," ACM International Symposium on Symbolic and Algebraic Computation (ISSAC), Maui (Hawaii, USA), July 1997.

[16] G. Caire, D. Tuninetti, "The Throughput of Hybrid ARQ protocols for the Gaussian collision channel," IEEE Transactions on Information Theory, vol. 47, no. 5, pp. 1971-1988, July 2001.

[17] S. Boyd and L. Vandenberghe, "Convex Optimization," Cambridge University Press, 2004.

[18] X. Lagrange, "Throughput of HARQ protocols on a block fading channel," IEEE Communications Letters, vol. 14, no. 3, pp. 257-259, March 2010

[19] D. Tse and P. Viswanath, "Fundamentals of Wireless Communications," Cambridge University Press, 2005.

[20] I.S. Gradshteyn and I.M. Ryzhik, "Table of Integrals, Series, and Products," Academic Press, 2007. 